\title{
Armut und Verteilung in Deutschland und Europa
}

Ute Klammer

\begin{abstract}
Scheinbar befinden wir uns in Deutschland zurzeit in einer günstigen Situation: Der Aufschwung ist unverkennbar, die registrierte Arbeitslosigkeit ist 2007 deutlich zurückgegangen. Allerdings entspricht dieser Aufwärtstrend nicht unbedingt der „gefühlten“ Entwicklung. Der Beitrag beleuchtet alte und neue Probleme im Bereich der Einkommensverteilung und Armut und ordnet die Befunde europäisch ein. Deutlich wird, dass die Europäische Union ihren hohen Ansprüchen hinsichtlich der Armutsbekämpfung und einer verbesserten sozialen Inklusion bisher nicht gerecht geworden ist.
\end{abstract}

\section{Armut und Verteilung im Schatten des Aufschwungs}

Die Medienberichterstattung wie auch die Vielzahl aktueller (wissenschaftlicher) Publikationen zeigt: Die ungleiche Einkommensverteilung, Armut und soziale Ausgrenzung sind (wieder) ein öffentlich diskutiertes Thema. Auch die neu aufgelebte Forderung nach Einführung eines bedingungslosen Grundeinkommens ist ein Indiz dafür, dass die Entwicklung der Einkommensverteilung und Teilhabechancen auf wachsende Kritik stößt. Ganz offensichtlich nimmt der gegenwärtige Aufschwung nicht alle mit, wir erleben derzeit keinen „Fahrstuhleffekt“.

Der Zweite Armuts- und Reichtumsbericht der Bundesregierung von 2005 (Bundesregierung 2005) hatte - auf Basis der Einkommens- und Verbrauchsstichprobe (EVS) - bereits einen Anstieg des relativen Armutsrisikos der Bevölkerung von 12,1\% auf 13,5\% zwischen 1998 und 2003 ausgewiesen. Als arm gelten Haushalte, die weniger als $60 \%$ des äquivalenz-gewichteten Medianeinkommens zur Verfügung haben. Weitere wichtige Ergebnisse waren:

- Das Armutsrisiko von Frauen und Männern gleicht sich an, allerdings durch ein überproportional gestiegenes Armutsrisiko von Männern.

- Es gibt hohe Armutsrisikoquoten für Kinder (15\% der unter 15-Jährigen), die den langfristigen Trend einer „Infantilisierung der Armut" in Deutschland bestätigen.

- Es gibt anhaltende, allerdings auf hohem Niveau stabilisierte Armutsprobleme von Alleinerziehenden sowie

- eine weiter (auf $24 \%$ ) gestiegene Armutsbetroffenheit von Migranten.
- Für Rentner zeigt sich dagegen bislang ein eher unterdurchschnittliches Armutsrisiko.

Auch bei der Vermögensverteilung sind Brennpunkte offensichtlich: Während der Anteil des Vermögens des wohlhabendsten Quintils der Bevölkerung in der Dekade zwischen 1993 und 2003 von 66,1\% auf $67,5 \%$ (leicht) angestiegen ist, weist das unterste Quintil der Bevölkerung inzwischen ein Negativvermögen, also Verschuldung, auf. Auch andere Indikatoren belegen einen - wenn auch geringfügigen Trend zu einer ungleichmäßigeren Einkommensverteilung. ${ }^{1}$

Neuere Daten des Sozio-ökonomischen Panels (SOEP) bestätigen das Bild (StBA 2006a, 2006b). Hier wird die allgemeine Armutsrisikoquote für 2004 auf $13 \%$, in Ostdeutschland sogar auf $17 \%$ beziffert. Angestiegen ist vor allem - schon vor den Hartz-Gesetzen - das Risiko, bei Arbeitslosigkeit zu verarmen. Galten definitionsgemäß 1997 nur 30,6 \% der Arbeitslosen als arm, so waren es 2004 schon $42 \%$. Insgesamt waren alleinlebende junge Erwachsene (53\%), Arbeitslose (43\%) sowie Alleinerziehende (30 \%) 2004 am häufigsten von Armut betroffen.

\section{Neue Armutsrisiken}

Neben die seit Langem bekannten Ursachen für Armutsgefährdung treten neue Risiken. Dazu zählen:

(1) Verringerter Schutz bei Arbeitslosigkeit: Die Arbeitslosenversicherung mit der vorgesehenen Lohnersatzleistung des ALG I (Arbeitslosengeld) erreicht - bei einer Querschnittsbetrachtung - einen zunehmend geringeren Anteil der Arbeitslosen.
Allein zwischen Anfang 2006 und Herbst 2007 hat sich der Anteil der Arbeitslosen, die ALG I beziehen, von über $30 \%$ auf unter $19 \%$ reduziert (o. V. 2007). Ursächlich dafür sind sowohl die verkürzte Bezugsdauer des ALG I als auch Veränderungen im Arbeitslosenbestand. Langzeitarbeitslose werden häufiger als früher vom Leistungsbezug ausgeschlossen, weil die Einkommen weiterer Mitglieder der häuslichen Bedarfsgemeinschaft stärker als früher angerechnet werden. Dass sich bei Arbeitslosigkeit das Verarmungsrisiko erhöht hat, macht die folgende Zahl deutlich: Fast jeder zehnte ALG I-Empfänger bezieht aufstockend ALG II. In dieser Entwicklung schlagen auch Niedrigeinkommen zu Buche, die bei Arbeitslosigkeit nur Arbeitslosengeldansprüche unterhalb des ALG IINiveaus nach sich ziehen.

(2) Armut trotz Arbeit: Eine in ihren Ausmaßen nicht vorausgeahnte Entwicklung ist im Anstieg der Zahl der sogenannten "Aufstocker" zu sehen, die trotz Arbeit auf ergänzenden ALG II-Bezug angewiesen sind (Trube/Wohlfahrt 2007). Anfang 2007 gingen bereits rund 1,28 Mio. Bezieher von ALG II einer bezahlten Beschäftigung nach, das war ein Viertel der erwerbsfähigen ALG II-Empfänger. Damit lag die Zahl der Aufstocker um 160.000 höher als noch im Oktober 2006. Mehr als 675.000 der auf zu-

\footnotetext{
1 So zeigt der GINI-Koeffizient zwischen 1998 und 2003 einen leichten Anstieg (von 0,255 auf $0,257)$
}

Ute Klammer, Prof. Dr., Universität DuisburgEssen. Arbeitsschwerpunkte: Europäische und vergleichende Wohlfahrtsstaatsforschung, Alterssicherung, Familienpolitik, Flexicurity. e-mail: ute.klammer@uni-due.de. 
sätzliche ALG II-Zahlungen angewiesenen Erwerbstätigen waren sozialversicherungspflichtig beschäftigt, 480.000 von ihnen sogar vollzeitbeschäftigt (BA 2007; Adamy 2007).

(3) Kinderarmut: Trotz der in jüngster Zeit bemerkenswerten „Renaissance“ der Familienpolitik, die durchaus mit Leistungsausdehnungen einhergeht und viele alte Forderungen (Elterngeld, Ausbau der Kinderbetreuung) anpackt, ist es bisher nicht gelungen, das Problem der Kinderarmut in den Griff zu bekommen. Dies verdeutlichen z. B. Zahlen aus dem „Kinderreport Deutschland 2007" (Deutsches Kinderhilfswerk 2007): Während in Deutschland 1965 nur jedes 75 . Kind unter sieben Jahren von Sozialhilfe lebte, war 2006 schon jedes sechste Kind betroffen. Gegenwärtig sind mehr als 2,5 Mio. Minderjährige auf Sozialgeld oder -hilfe angewiesen. Das entspricht einer Verdopplung seit Inkrafttreten von Hartz IV und einem Anstieg von 100.000 im Verlauf des Jahres 2007 - trotz der verbesserten Konjunktur. Im Juni 2007 lebten knapp 17 \% aller Kinder in Deutschland in Familien, die Leistungen nach dem SGB II erhielten; in Ostdeutschland waren es sogar $31 \%$. Besonders häufig sind Migrantenkinder betroffen.

(4) Altersarmut: Ein Problem, das in der Öffentlichkeit erst seit Kurzem wahrgenommen wird, ist die absehbare Rückkehr der Altersarmut. Aufgrund der seit 2001 beschlossenen Reformen im Alterssicherungsbereich ist das Rentenniveau für den sogenannten „Eckrentner“ mit 45 Versicherungsjahren bzw. Entgeltpunkten bereits auf $63 \%$ des Nettoeinkommens gesunken und wird in den nächsten 30 Jahren weiter bis auf ca. $43 \%$ absinken. Neue erwerbsbiografische Risiken lassen zudem erwarten, dass Männer der Geburtsjahrgänge 1942-1961 - so die Vorausschätzungen der Ende 2007 vorgestellten Studie „Altersvorsorge in Deutschland (AVID)“ - nur durchschnittlich 38 Versicherungsjahre erreichen werden, Frauen 33 Jahre. Die Einschnitte können die heutigen Erwerbstätigen selbst bei 100 \%iger Ausnutzung der Riester-Rente voraussichtlich nicht vollständig ausgleichen. Nach offizieller Darstellung (Rische 2007) werden Personen der Jahrgänge 1957-1961 ohne private Vorsorge ceteris paribus $88 \%$ des Versorgungsniveaus der 1942-1946 Geborenen erreichen, mit Riester-Vorsorge (bei einer ange- nommenen Verzinsung von $2,75 \%$ und $100 \%$ iger Teilnahme an der privaten Vorsorge) $95 \%$ in Westdeutschland, im Osten $93 \%$. Die Gefahr der Altersarmut wird vor allem in den Neuen Bundesländern zunehmen. Bei diesen Prognosen sind allerdings zukünftige verminderte Rentenanpassungen, wie sie angesichts des bereits beschlossenen Nachhaltigkeitsfaktors zu erwarten sind, noch ebenso wenig berücksichtigt wie die steigende Steuerbelastung von Rentnern durch den sukzessiven Übergang zur nachgelagerten Besteuerung. Ein nochmals besonderes Risiko, im Alter zu verarmen, haben Personen mit längeren Arbeitslosigkeitsphasen, geringfügiger Beschäftigung und Kleinselbstständige.

Als Zwischenfazit lässt sich festhalten, dass in Deutschland unter der trügerisch "glatten“ Oberfläche einer langfristig nur wenig ungleicher gewordenen Einkommensverteilung problematische Entwicklungen für bestimmte Bevölkerungsgruppen zu beobachten sind. Diese deutsche Entwicklung fügt sich in das Gesamtbild der europäischen Entwicklungen ein, selbst wenn es Abweichungen zwischen einzelnen Ländern gibt (vgl. Abschnitt 4). Was sich hier abzeichnet, steht im Kontrast zum erklärten EU-Ziel, Armut und soziale Exklusion zu bekämpfen.

\section{Armutsbekämpfung und soziale Eingliederung in der Europäischen Sozialpolitik}

Die Bekämpfung von Armut ist eines der ältesten Ziele der EU-Sozialpolitik. Dieser Aufgabe hatte sich die Europäische Gemeinschaft bereits vor Jahrzehnten verpflichtet, in einer Zeit, in der Sozialpolitik ansonsten noch - dem Subsidiaritätsprinzip verpflichtet - als eigenständige Aufgabe der Mitgliedsländer betrachtet wurde. In ihrer heutigen Form hat die Bekämpfung von Armut und sozialer Ausgrenzung durch die EU ihre Grundlage im Vertrag von Amsterdam (Art. 136 und 137). Auf der Tagung des Europäischen Rats in Nizza Ende 2000 wurde die Entscheidung von Lissabon im März 2000 bekräftigt und umgesetzt, die Methode der offenen Koordinierung (MOK) auch als Grundlage für den Kampf gegen Armut und soziale Ausgrenzung zu nutzen. Zentrale Elemente dieses Ansatzes sind: die Festlegung ge- meinsamer Ziele für die EU als Ganzes, die Ausarbeitung geeigneter Nationaler Aktionspläne zur Erreichung der Ziele sowie die regelmäßige Berichterstattung und Überwachung der Fortschritte.

Im Dezember 2001 einigte sich der Europäische Rat in Laeken auf zunächst 18 gemeinsame statistische Indikatoren (Eurostat 2004, S. 1, 8; StBA 2006a, S. 43f.), darunter zahlreiche Armutsindikatoren, die es ermöglichen sollen, die Fortschritte der Mitgliedstaaten in Richtung einer verbesserten sozialen Integration zu überprüfen. Seit 2006 wird eine „integrierte MOK“ im Bereich Sozialschutz und Eingliederung durchgeführt, die die MOK der Bereiche soziale Eingliederung, Renten, Gesundheitsversorgung und Langzeitpflege zusammengeführt hat. Dabei wurden für den Arbeitsbereich „Beseitigung von Armut und sozialer Ausgrenzung " anspruchsvolle Ziele formuliert, die über die materielle Dimension von Armut weit hinausgehen. Die Zielsetzungen haben im Blick, dass die angestrebte soziale Integration den freien $\mathrm{Zu}$ gang für alle zu allen notwendigen gesellschaftlichen Ressourcen, Rechten und Dienstleistungen ebenso voraussetzt wie den Kampf gegen alle Formen von Diskriminierung. Sie reflektieren auch, dass eine solche Politik breite Unterstützung durch die Regierungen und die gesellschaftlichen Akteure erfordert. Es handelt sich um ein Programm, das eng mit der Lissabon-Strategie verzahnt ist und zudem einen Schwerpunkt auf „Governance“ und die Beteiligung der unterschiedlichen Akteure legt. Inzwischen haben die Mitgliedstaaten erstmals integrierte nationale Berichte über ihre Strategien in den Bereichen soziale Eingliederung, Renten, Gesundheitsversorgung und Langzeitpflege vorgelegt.

\section{Armut und Einkommens- verteilung in der EU - Zahlen und Fakten}

\subsection{ARMUTSINDIKATOREN}

Von 1994-2001 war das European Community Household Panel (ECHP) die Hauptdatenquelle für die vergleichende Berechnung von Armut und Einkommen in der EU. 2003 wurde der Übergang zu einer neuen Datenbasis, der Community Statistics on Income and Living Conditions (EU- 
SILC), beschlossen. EU-SILC dient der Erhebung aktueller und vergleichbarer multidimensionaler Quer- und LängsschnittMikrodaten über Einkommen, Armut, soziale Ausgrenzung und Lebensbedingungen. Die Erhebung von EU-SILC-Daten ist 2004 in 13 EU-Mitgliedstaaten (plus Norwegen und Island) angelaufen, die übrigen Länder beteiligen sich seit 2005. Ein erster Satz von EU-SILC Mikrodaten und Querschnittsindikatoren für alle EU25-Mitgliedstaaten liegt seit Ende 2006 vor (StBA 2006a, S. 37-40; Eurostat 2005a, S. 1).

(1) Betrachtet man die Beschäftigtenquoten und den Rückgang der Arbeitslosigkeit als maßgebliche Indikatoren für die Bekämpfung von Armut, so lässt sich für die EU neuerdings eine durchweg positive Entwicklung konstatieren: Zwischen Sommer 2006 und 2007 hat sich EU-weit die Erwerbstätigenquote um rund einen Prozentpunkt gesteigert, und zwar bei Männern wie bei Frauen; es gab einen deutlichen Rückgang der Erwerbslosenquote und einen (geringeren) Rückgang der Langzeitarbeitslosenquote. Die Arbeitslosenquote lag (September 2007) in der EU27 bei $7 \%$. Deutschland lag mit 8,1\% oberhalb des Durchschnitts und in der Gruppe von nur sieben Ländern, die mehr als 8 \% Arbeitslosigkeit auswiesen. Nach den Schätzungen von Eurostat waren im September 2007 noch etwa 16,7 Mio. Menschen in der EU27 arbeitslos (Sept. 06: 18,8 Mio.). Den Prognosen des Berichts „Beschäftigung in Europa 2007" zufolge (Europäische Kommission 2007a) soll die Arbeitslosenquote 2008 sogar auf $6,7 \%$ sinken.

(2) Working Poor: Eine nur spärliche Datenlage gibt es im EU-Vergleich für das Problemfeld der „Working Poor“. Gemeint sind Menschen, die trotz Erwerbsarbeit arm sind. Eine Eurostat-Untersuchung (Eurostat 2005b) ermittelte, dass $20017 \%$ der Erwerbstätigen in der EU15 Einkommen unterhalb der Armutsgrenze hatten. Besonders hoch - mit $10 \%$ oder mehr - lag das Armutsrisiko der Erwerbstätigen in den vier Ländern der EU-Südschiene (Spanien, Italien, Portugal und Griechenland). Deutschland (4\%) gehörte neben Dänemark, Belgien und Schweden zur Gruppe der Länder mit dem geringsten Armutsrisiko für Erwerbstätige. Im EU-Durchschnitt hatten Selbstständige ein 2,5fach höheres Armutsrisiko als abhängig Beschäftigte (Eurostat 2005b; European
Foundation 2004, 2007). Hierbei ist zu berücksichtigen, dass die Zahlen nicht die Anteile der Niedriglöhner wiedergeben, sondern die Einkommenssituation des jeweiligen Haushalts.

(3) Einkommensverteilung: Dem „S80/S20“Indikator von Laeken zufolge hatten die reichsten $20 \%$ der Bevölkerung in der EU 2003 durchschnittlich das 4,6-fache Einkommen der ärmsten $20 \%$. Besonders ausgeprägt war die Ungleichverteilung des Einkommens in Portugal $(7,4)$, besonders gleichmäßig in Ungarn $(3,0)$. Der GINIKoeffizient wurde mit $29 \%(0,29)$ berechnet und bewegte sich zwischen $22 \%$ für Slowenien und $35 \%$ im Vereinigten Königreich und in Griechenland. Insgesamt ist die Streuung der Einkommen bisher in den meisten neuen Mitgliedstaaten (NMS) niedriger als im Durchschnitt der EU15, d.h. die Ungleichverteilung ist dort bisher noch nicht so ausgeprägt.

(4) Armut: Gemessen an der Armutsschwelle (60\% des Medianeinkommens) waren $200316 \%$ der EU-Bevölkerung von Armut bedroht; dies entsprach einer Zahl von rund 72 Mio. Personen. Die höchsten Armutsquoten wiesen die Slowakei, Irland und Griechenland auf (21\%), die niedrigsten mit Werten um $10 \%$ die Tschechische Republik, Luxemburg, Ungarn und Slowenien (Eurostat 2005a; Europäische Kommission 2007b). In den meisten EU-Ländern haben Kinder ein überdurchschnittliches Armutsrisiko, in einigen Ländern ist nahezu jedes dritte Kind betroffen. Die Interpretation dieser Daten verändert sich allerdings, wenn man in Rechnung stellt, dass in einigen Mitgliedsländern - so beispielsweise bei den Neumitgliedern - die nationalen Armutsschwellen sehr viel niedriger liegen als in Vergleichsländern, womit auch die Lebensbedingungen entsprechend schlechter sind. So zeigt sich bei einer Umrechnung in Kaufkraftstandards, dass in den Ländern mit der niedrigsten Armutsgefährdung tendenziell die höchsten Armutsschwellen gelten und umgekehrt. Neun der zehn im Jahr 2004 beigetretenen Mitgliedstaaten weisen eine Armutsschwelle aus, die unter dem EU25-Durchschnittswert liegt (Eurostat 2005a, S. 1, 8).

Da Einkommensindikatoren also nur begrenzt Aufschluss über die tatsächlichen Lebensbedingungen der Armen geben, hat Eurostat ergänzende Daten erhoben, um die materiellen Lebensbedingungen bzw. Formen „materieller Entbehrung“ insgesamt erfassen zu können (Eurostat 2005a). Sie verdeutlichen u. a., dass 2003 in fünf der zehn im Jahr 2004 beigetretenen neuen Mitgliedstaaten 30 \% der Bevölkerung Einschränkungen in der Ernährung hinnehmen müssen (wie z. B. nur gelegentlicher Genuss von Fisch und Fleisch) oder dass in den baltischen Staaten rund $20 \%$ der Bevölkerung benachteiligte Wohnverhältnisse haben (z. B. kein in der Wohnung gelegenes WC). Verknüpft man die Ergebnisse zum Armutsrisiko (gemessen an der $60 \%$ Schwelle des Medianeinkommens) mit solchen Deprivationsindikatoren (Intersection-Methode), so zeigt sich, dass Armut das Deprivationsrisiko in Bezug auf einen Mangel an Gebrauchsgütern um das Dreifache, in Bezug auf ökonomische Anspannung um das Zweifache und in der Dimension „Wohnen“ um einen Faktor von mehr als 1,5 erhöht (ebd.). Hier wird anschaulich, dass der Vergleich von Armut und sozialer Ausgrenzung in den EU-Staaten angesichts des großen Wohlfahrtsgefälles zwischen den Ländern weit über die Bemessung der relativen Armut und auch über die ursprünglichen Laeken-Indikatoren hinausgehen muss.

\subsection{ARMUTSENTWICKLUNC}

Folgt man den von Eurostat bereitgestellten Daten, so lassen sich - auch wenn nicht für alle Indikatoren und Länder lange Reihen zur Verfügung stehen - folgende Erkenntnisse hinsichtlich der Entwicklung der Armut und Einkommensverteilung gewinnen (http://epp.eurostat.ec.europa.eu):

(1) Zwischen 1996 und 2005 ist die Einkommensverteilung gemessen an dem Indikator S80/S20 in der EU15 weitgehend konstant geblieben. In der EU25 dagegen zeigt sich eine Zunahme der Ungleichverteilung (von 4,6 1998 auf 4,9 2005). Insgesamt sind die Entwicklungen auch hier uneinheitlich. Eine klare Zunahme der Einkommensungleichheit weisen im Beobachtungszeitraum z. B. Dänemark und das Vereinigte Königreich aus, während die Zahlen für Griechenland, Spanien, Frankreich, Luxemburg eher auf eine gleichmäßiger werdende Einkommensverteilung hinweisen.

(2) Die Armutsrisikoquote - gemessen an $60 \%$ des nationalen Median-Einkommens- 
lag 2005 in der EU15 mit 16 \% genauso hoch wie 1996. Ähnliche Werte werden seit 1998 regelmäßig für die EU25 ausgewiesen. In den meisten Ländern war in der letzten Dekade den Eurostat-Daten zufolge keine deutliche Veränderung des Armutsrisikos festzustellen. Dies gilt allerdings nicht für die EU-Neumitglieder Lettland, Litauen und Polen sowie für Finnland, wo die Armutsquote deutlich zunahm. Rückläufige Armutsrisiken verzeichneten dagegen z. B. Portugal und auch Frankreich.

(3) Für Kinder und Jugendliche wiesen 2005 alle EU-Länder hohe Armutsrisikoquoten im zweistelligen Bereich auf; in sieben Mitgliedstaaten (vor allem in den NMS, aber auch im Vereinigten Königreich) lagen die entsprechenden Quoten sogar über $20 \%$. Dennoch kann im EU-Durchschnitt (18 \%) auch hier in der letzten Dekade von einer „Stabilität auf hohem Niveau" gesprochen werden. Weiter gestiegen ist das Armutsrisiko für Kinder vor allem in einigen neuen Mitgliedstaaten; leicht rückläufige Werte werden für einige alte Mitgliedstaaten ausgewiesen.

(4) Alleinerziehende gehören überall in der EU zu den besonders von Armut betroffenen Gruppen, unter ihnen ist durchschnittlich ein Drittel einkommensarm. Jedoch sind die Tendenzen in Bezug auf diese Gruppe stark gegenläufig: Während in einigen Ländern (z. B. Niederlande, Österreich, Vereinigtes Königreich, auch Deutschland) das Armutsrisiko für Alleinerziehende verringert werden konnte, hat sich die Situation in anderen Ländern (z. B. Griechenland, Italien, Litauen, Finnland) teilweise eklatant verschlechtert. Am besten vermögen heute Schweden und Dänemark das Armutsrisiko von Alleinerziehenden zu begrenzen.

(5) Bei der Entwicklung der Altersarmut zeigen sich zwischen den Ländern, aber auch im Zeitverlauf, gewaltige Differenzen. 2005 bewegte sich das Armutsrisiko für die ältere Bevölkerung - Personen ab 65 Jahren - in den EU-Ländern zwischen $5 \%$ (Niederlande) und $51 \%$ (Zypern). In der EU15 ist das durchschnittliche Risiko der Altersarmut mit $20 \%$ in der letzten Dekade etwa stabil geblieben, in der EU25 geringfügig von $18 \%$ auf $19 \%$ gestiegen. Verbesserungen für Rentner waren vor allem in Österreich, Griechenland, Dänemark und auch Belgien zu verzeichnen, während die Al-

Tabelle 1: Ranking der EU-Länder in Bezug auf ausgewählte Indikatoren

\begin{tabular}{|c|c|c|c|c|c|}
\hline & $\begin{array}{l}\text { Ungleichheit } \\
\text { der } \\
\text { Einkommens- } \\
\text { verteilung } \\
\text { (S80/S20) } \\
2003\end{array}$ & $\begin{array}{l}\text { Armutsrisiko- } \\
\text { quote ( } 60 \% \\
\text { des Medians) } \\
\text { nach sozialen } \\
\text { Transfers } \\
2003\end{array}$ & $\begin{array}{l}\text { Netto- } \\
\text { Lohnersatzrate } \\
\text { bei Arbeits- } \\
\text { losigkeit, erste } \\
60 \text { Monate } \\
2004\end{array}$ & $\begin{array}{l}\text { Minderjährige } \\
\text { Kinder in } \\
\text { Erwerbslosen- } \\
\text { haushalten } \\
2005\end{array}$ & $\begin{array}{l}\text { Armutsrisiko- } \\
\text { quote (60\% } \\
\text { des Medians) } \\
\text { für Personen } \\
\text { ab } 65 \text { Jahren } \\
2003\end{array}$ \\
\hline $\begin{array}{l}\text { Durchschnitts- } \\
\text { wert EU }\end{array}$ & 4,6 & $15 \%$ & $62 \%$ & $9,6 \%$ & $17 \%$ \\
\hline Wert Deutschland & 4,3 & $15 \%$ & $75 \%$ & $10,9 \%$ & $16 \%$ \\
\hline Rang Deutschland & 12 & 10 & 5 & 18 & 10 \\
\hline Bestes Land & $\begin{array}{l}\text { Slowenien } \\
(3,1)\end{array}$ & $\begin{array}{l}\text { Tschechische } \\
\text { Republik (8\%) }\end{array}$ & $\begin{array}{l}\text { Niederlande } \\
(79 \%)\end{array}$ & $\begin{array}{l}\text { Slowenien } \\
(2,7 \%)\end{array}$ & $\begin{array}{l}\text { Tschechische } \\
\text { Republik (4\%) }\end{array}$ \\
\hline Schlechtestes Land & $\begin{array}{l}\text { Portugal } \\
(7,6)\end{array}$ & $\begin{array}{l}\text { Griechenland/ } \\
\text { Slowakei/ } \\
\text { Irland (21 \%) }\end{array}$ & Italien $(6 \%)$ & $\begin{array}{l}\text { Vereinigtes } \\
\text { Königreich } \\
(16,5 \%)\end{array}$ & Zypern (52\%) \\
\hline \multicolumn{6}{|c|}{ Rankings umfassen - je nach Datenverfügbarkeit - 19 bis 23 EU-Länder. } \\
\hline \multicolumn{6}{|c|}{$\begin{array}{l}\text { Quelle: Zusammenstellung der Autorin auf Basis Jörgensen/Schulz zur Wiesch 2006, } \\
\text { S. 12-16, Eurostat-Daten. }\end{array}$} \\
\hline
\end{tabular}

tersarmut in den mediterranen Ländern Spanien, Italien, Portugal wie auch in Finnland deutlich zugenommen hat.

(6) Bezüglich des Indikators dauerhafte Armutsgefährdung ist die Datenlage lückenhaft, jedoch sind auch hier die Differenzen zwischen den Ländern viel deutlicher als die Veränderungen in den einzelnen Ländern im Zeitablauf. Mit einer Quote von $6 \%$,dauerhaft“ (d.h. in mehreren aufeinander folgenden Jahren) armen Personen lag Deutschland 2001 bei den Ländern mit den günstigsten Werten. Allerdings hat sich die Quote in Deutschland bis 2004 - also innerhalb kürzester Zeit - auf $9 \%$ (Frauen: $11 \%$ ) erhöht - ein Beleg dafür, dass es inzwischen schwieriger als noch vor einigen Jahren ist, aus der Armut wieder herauszukommen.

Insgesamt bestätigen die Eurostat-Daten nicht den Eindruck, dass Armut und Einkommensungleichheiten allgemein und deutlich zunehmen. Vielmehr sind die Ergebnisse in Bezug auf die ausgewiesenen Indikatoren und Länder recht heterogen. Dies lässt allerdings die Fragen unberührt, ob die europaweit erhobenen Indikatoren die Entwicklungen differenziert genug abbilden und ob die MOK einen wesentlichen Einfluss auf die Entwicklungen hatte.

\subsection{SOZIALE SICHERUNG - SCHUTZ VOR ARMUT?}

In allen EU-Ländern tragen die sozialen Sicherungssysteme zur Verminderung des Armutsrisikos bei. Würden sämtliche Sozialtransfers wegfallen, hätte die Armutsrisikoquote in der EU ceteris paribus $200340 \%$ statt $16 \%$ betragen, betrachtet man Ren- tenleistungen als aufgeschobenes Erwerbseinkommen und rechnet sie aus den Sozialleistungen heraus, hätte die Armutsquote vor Sozialleistungen immer noch bei $25 \%$ gelegen. An der Spitze bezüglich der Armutsreduktion durch Sozialleistungen liegen die skandinavischen Sozialleistungssysteme. Deutschland lag 2003 leicht über dem EU25-Durchschnitt im Mittelfeld (Eurostat 2005a). Durchschnittlich machten die Sozialschutzausgaben 2004 in der EU 27,3 \% des BIP aus. Allerdings ist beim Sozialschutz in der EU ein großes Gefälle zu konstatieren (Eurostat 2007b). So liegt die Sozialleistungsquote in einigen Ländern mehr als doppelt so hoch wie in anderen. In Kaufkraftparitäten pro Kopf gerechnet geben einige Länder - wie die baltischen Staaten - sogar sieben Mal weniger für soziale Sicherheit aus als die drei EULänder mit den höchsten Sozialschutzausgaben. Angesichts der Entwicklungen der vergangenen Jahre erscheint es fraglich, ob die neuen EU-Mitgliedstaaten bezüglich des Sozialschutzes so aufholen werden, wie dies bei früheren EU-Erweiterungsrunden zu beobachten war.

Obgleich Deutschland im EU-Vergleich eines der Länder mit der höchsten Sozialleistungsquote ist, steht es in Bezug auf verschiedene Armuts- und Verteilungsindikatoren sowie die Erreichung wichtiger sozialer Ziele keineswegs auf einem Spitzenplatz (Tabelle 1). So findet sich Deutschland bei einem aktuellen Ranking der EULänder von Jörgensen und Schulz zur Wiesch (2006) zu verschiedenen sozialen Aufgabenfeldern in der Dimension „Einkommensverteilung und soziale Sicherung" im EU-Vergleich auf Platz neun, in der Dimension „Inklusion in den Arbeitsmarkt" ebenso wie bei den „Bildungs- und 
Ausbildungschancen“ auf Platz 18, bezüglich der "Geschlechtergleichstellung" und dem „Generationenverhältnis“ sogar nur auf Platz 23.

\section{Bilanz und Perspektiven}

Auch wenn die Eurostat-Daten den vermuteten deutlichen Anstieg der Armut in Europa nicht bestätigen, so weisen sie doch aus, dass die EU im Bereich der Armutsbekämpfung bisher - ungeachtet der Steigerung der Beschäftigungsquoten - wenig erfolgreich war. Dies gilt erst recht, wenn man die lange Tradition berücksichtigt, die die Armutsbekämpfung im Unterschied zu anderen sozialpolitischen Feldern in der EU hat.

Mit der Ausdehnung der Methode der offenen Koordinierung (MOK) auf den Bereich Armut/Soziale Inklusion hat die EU aber immerhin neue Möglichkeiten, auf Missstände hinzuweisen und die Länder aufzufordern, in bestimmten Feldern stärker aktiv zu werden. Bereits jetzt haben die europäischen Benchmarks auf Nachholbedarf wiederholt aufmerksam gemacht, so beispielsweise darauf, dass Deutschland bei der Betreuungsquote der Unter-Dreijährigen oder bei der Beschäftigungsquote älterer Arbeitnehmer deutlich unterhalb der EU-Ziele liegt - was hierzulande in beiden Feldern neue Anstrengungen entfacht hat. Die Potenziale des gegenseitigen Lernens sollten also nicht unterschätzt werden.

Die neue, ,integrierte“ MOK kann den Blick auf die Zusammenhänge weiter verbessern. Bereits der erste vergleichende Bericht hat auf die steigende Ungleichheit in vielen Gesundheitssystemen (Zunahme von Zuzahlungen, Eigenleistungen) und die Folgen dieser Entwicklung für arme und benachteiligte Bevölkerungsgruppen (Zugangsbeschränkungen, Trend zur Ungleichverteilung) hingewiesen.

Dessen ungeachtet ist die Frage weiterzuverfolgen, ob und wie der MOK mehr Nachdruck in Bezug auf die Anstrengungen der Mitgliedstaaten zur Armutsbekämpfung verliehen werden kann. $\mathrm{Ob}$ dies über eine Verstärkung der Zusammenarbeit von Gruppen von Mitgliedstaaten in einem Europa unterschiedlicher Geschwindigkeiten erfolgen kann oder ob die Kopplung der MOK mit „hard law“ und entsprechenden Sanktionsmöglichkeiten möglich sein wird (Scharpf 2002), muss geprüft werden.

In Deutschland haben einige Reformen der letzten Jahre gezielt an den Armutsproblemen bestimmter Gruppen angesetzt: So konnte durch die Einführung einer bedarfsorientierten Mindestsicherung im Alter das Problem der verschämten Armut und Nicht-Inanspruchnahme von Sozialleistungen durch ältere Bedürftige reduziert werden; das Armutsrisiko Alleinerziehender konnte, wenn auch auf hohem Niveau, zumindest stabilisiert werden. Und zuletzt hat die anziehende Konjunktur einem Teil der Arbeitslosen den Weg aus der Arbeitslosigkeit und Armut (vorübergehend?) geebnet. Jedoch haben andere Reformen - wie die Einführung des ALG II neue Armutsprobleme nach sich gezogen oder werden sie - wie die Rentenreformen seit 2001 - absehbar in der Zukunft nach sich ziehen.

Bei der Diskussion zukünftiger Strategien zur Bekämpfung von Armut und sozialer Ausgrenzung darf die Bindung an die EU-Sozialpolitik nicht ignoriert werden, deren Credo unverkennbar ist: Sozialpolitische Maßnahmen sollen vor allem das Wirtschafts- und Beschäftigungswachstum fördern, um einen Beitrag zum sozialen Zusammenhalt zu leisten. So liegen dann auch Vorschläge, die auf ein bedingungsloses Grundeinkommen hinauslaufen - ungeachtet anderer fragwürdiger Aspekte (wie Finanzierbarkeit, Anrecht auf Teilhabe an der Erwerbsgesellschaft, Geschlechterverhältnis, Bewertung unterschiedlicher Formen von Nicht-Erwerbsarbeit etc.) quer zu den Zielen der Europäischen Beschäftigungsstrategie.

Als wichtigster Ansatzpunkt bei der Bekämpfung von Armut und sozialer Ausgrenzung kann die Verbesserung der Primärverteilung angesehen werden, da der Sozialstaat mit dem Ausgleich einer ungleicher werdenden Verteilung der Erwerbseinkommen zunehmend überfordert ist.
Hier ist eine Umkehr von der Förderung geringfügiger Beschäftigung ebenso erforderlich wie der weitere Einsatz für Mindestlöhne, auch wenn Mindestlöhne allein, wie sich im EU-Vergleich zeigt (European Foundation 2007, S. 9), nicht Garant für niedrige Armutsquoten sind.

Eine wichtige Rolle bei der Bekämpfung von Kinder- und Familienarmut kommt einem verbesserten Familienlastenausgleich zu. Die geplante Ausweitung des anspruchsberechtigten Personenkreises für den 2005 eingeführten, komplizierten Kinderzuschlag (vgl. den Beitrag von Becker in diesem Heft) ist hier ein richtiger Schritt, der allerdings für eine Kindergrundsicherung noch nicht ausreichen dürfte. Im Hinblick auf die Verwirklichungschancen von Kindern muss weiterhin auch der Ausbau von Sozialdienstleistungen, z. B. der Kinderbetreuung, im Fokus stehen. Dies entspricht dem von der Kommission beschriebenen und empfohlenen Policy-Mix in diesem Feld (Europäische Kommission 2007b, S. 6).

Probleme der Armut im Alter können teilweise durch eine Verlängerung der Lebensarbeitszeit aufgefangen werden. Dies setzt allerdings neben einer weiteren Verbesserung der Arbeitsmarktlage eine nachhaltigere Nutzung von Arbeitskraft als bisher voraus. Um Lücken in den Versicherungsbiografien zu minimieren, dürfte zudem die Einführung einer allgemeinen Versicherungspflicht in der GRV unerlässlich sein, flankiert von mindestsichernden Ausgleichselementen für Personen mit besonders schwachen Versicherungsverläufen.

Abschließend ist auf die Schlussfolgerungen aus dem ersten Armutsprogramm der Europäischen Gemeinschaft (1981) zu verweisen, die auch nach einem Vierteljahrhundert nicht an Relevanz verloren haben:

„Würden die Mitgliedstaaten beim Kampf gegen die Armut zusammenstehen, würde der Fortschritt weniger durch die Angst um die Erhaltung der Wettbewerbsfähigkeit gehemmt werden " (Kommission der Europäischen Gemeinschaften 1981, S. 160). 
Adamy, W. (2007): Neue Untersuchungen zu Geringverdienern mit aufstockendem ALG II. 1,2 Millionen können vom Arbeitseinkommen nicht leben, in: Soziale Sicherheit 56, S. 180-189

Bundesagentur für Arbeit (BA) (2007): Anrechenbare Einkommen und Erwerbstätigkeit, August, Nürnberg

Bundesregierung (2005): Lebenslagen in Deutschland. Der 2. Armutsund Reichtumsbericht der Bundesregierung, Berlin

Deutsches Kinderhilfswerk (Hrsg.) (2007): Kinderreport Deutschland 2007, Freiburg

Europäische Kommission (2007a): Beschäftigung in Europa 2007, Luxemburg

Europäische Kommission (2007b): Mitteilung der Kommission vom

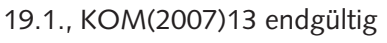

European Foundation (2004): Working poor in the European Union, Dublin/Luxemburg

European Foundation (2007): Income Poverty in the European Union, Dublin

Eurostat (2004): Armut und soziale Ausgrenzung in der EU, Statistik kurz gefasst 16

Eurostat (2005a): Einkommensarmut und soziale Ausgrenzung in EU-25, Statistik kurz gefasst 13

Eurostat (2005b): In-Work Poverty, Statistics in focus 5

Eurostat (2007a): Euro-Indikatoren, Pressemitteilung 149 vom 31.10.

Eurostat (2007b): Sozialschutz in der Europäischen Union, Statistik kurz gefasst 99

Hauser, R. (2007): Anmerkungen zur Untersuchung "Alterssicherung in Deutschland“" Vortrag gehalten auf der Tagung der HBS und des DGB zum Thema "Sozialpolitische Herausforderungen in der Alterssicherung gestalten“", Berlin 22.11., unveröffentlichtes Manuskript
Hock, B./Holz, G./Simmedinger, R./Wüstendörfer, W. (2000): Gute Kindheit - schlechte Kindheit. Armut und Zukunftschancen von Kindern und Jugendlichen in Deutschland, Frankfurt a. M.

Jörgensen, J. F./Schulz zur Wiesch, J. (2006): Wie Sozial ist Europa? Eine Kurzstudie zur Sozialen Lage in der EU, Düsseldorf/Berlin, online: http://epp.eurostat.ec.europa.eu, Zugriff am 15.11.2007

Kommission der Europäischen Gemeinschaften (1981): Schlussbericht von der Kommission an den Rat über das Erste Programm von Modellvorhaben zur Bekämpfung der Armut, Brüssel

Rische, H. (2007): Vorstellung der Studie "Altersvorsorge in Deutschland (AVID) “ ${ }^{\prime}, 3$. Aktuelles Presseseminar der Deutschen Rentenversicherung Bund, Würzburg 20.-21.11., online: www.deutsche-rentenversicherung.de, Zugriff am 25.11.2007

Scharpf, F. W. (2002): The European Social Model: Coping with the Challenges of Diversity, in: Journal of Common Market Studies 4, S. 645-670 Statistisches Bundesamt (StBA) (2006a): Armut und Lebensbedingungen. Ergebnisse aus LEBEN IN EUROPA für Deutschland 2005, Wiesbaden

Statistisches Bundesamt (StBA) (Hrsg.) (2006b): Datenreport 2006, Bonn Taskforce Beschäftigung (2003), Jobs, Jobs, Jobs. Mehr Beschäftigung in Europa schaffen. Bericht der Taskforce Beschäftigung,Brüssel, online: http://www.eu.int/comm/employment_social/employment_strategy/ pdf/etf_de.pdf, Zugriff am 10.2.2006

Trube, A./Wohlfahrt, N. (2007): Arbeit und Armut - Die deutsche Unterschichtsdebatte im Lichte aktivierender Sozialstaatlichkeit, in: Gesundheits- und Sozialpolitik 61, S. 50-58

o. V. (2007): Verarmungsrisiko bei ALG I, in: Einblick 21, S. 3 